\title{
Terapia para la Eficacia Social y Tratamiento de Adultos-Jóvenes Españoles con Fobia Social Generalizada
}

\author{
Social Effectiveness Therapy for the Treatment of Young Spanish Adults with \\ Generalized Social Phobia
}

\author{
Pablo Olivares-Olivares \\ Universidad de Murcia, España \\ Diego Macià \\ Universidad Miguel Hernández de Elche, España \\ José Olivares \\ Universidad de Murcia, España
}

(Rec: 16 de noviembre de 2011 / Acep: 11 de junio de 2012)

\begin{abstract}
Resumen
El propósito de este estudio es adaptar y validar para su aplicación en población adulta española el tratamiento Terapia para la Eficacia Social (Turner, Beidel, Cooley, Woody y Messer, 1994). Se presentan los resultados obtenidos con un grupo experimental-tratamiento compuesto por 16 participantes que fueron comparados con un grupo de control-lista de espera, todos con fobia social generalizada (APA, 2000). La evaluación se realizó antes y después del tratamiento en ambos grupos, así como en dos medidas de seguimiento en el grupo experimental.

Los resultados en el grupo experimental muestran la eficacia a corto y a largo plazo de la adaptación realizada de la Terapia para la Eficacia Social en todas las medidas que evalúan la ansiedad y evitación social; por el contrario, los integrantes del grupo de control no alcanzaron mejoría en ninguna de las variables evaluadas. Palabras clave: fobia social generalizada, adultos jóvenes, Terapia para la Eficacia Social, tratamiento en grupo, experimental.
\end{abstract}

\begin{abstract}
The objective of this study is to adapt and validate for use in the adult Spanish population the treatment known as social effectiveness therapy (Turner, Beidel, Cooley, Woody, and Messer, 1994). We present results that were obtained by comparing an experimental-treatment group consisting of 16 participants with a waiting list control group, all with generalized social phobia (APA, 2000). The evaluation was carried out before and after the treatment in both groups as well as in two follow-ups with the experimental group.

The results in the experimental group show the short and long-term efficacy of social effectiveness therapy for all measures that assess social anxiety and avoidance. In contrast, the members of the control group did not experience improvement in any of the variables evaluated.

Keywords: generalized social phobia, young adults, Social Effectiveness Therapy, treatment group, experimental.
\end{abstract}

Correspondencia: Departamento de Personalidad, Evaluación y Tratamiento Psicológicos, Facultad de Psicología, Campus de Espinardo, Universidad de Murcia. Apdo. 4021, 30100 Murcia (España).E-mail: jorelx1@um.es. Teléfono: 868883976. 


\section{Introducción}

La fobia social, también denominada trastorno de ansiedad social, se caracteriza por un miedo acusado y persistente a una o más situaciones sociales o actuaciones en público en las que la persona se expone a la observación y a la evaluación negativa por parte de otros (APA, 2000). Estas situaciones sociales pueden ser muy variadas: hablar ante figuras de autoridad, ir a fiestas, establecer citas con otras personas, hablar ante una audiencia, etc. La exposición a estos estímulos produce una respuesta inmediata de ansiedad. El temor generado por tales situaciones, que la persona reconoce como irracional o desproporcionado, le lleva a evitarlas, lo que termina interfiriendo de forma notoria en sus actividades diarias, relaciones laborales y vida social, o produciéndole un malestar clínicamente significativo.

A la luz de los datos disponibles, se estima que entre el $7 \%$ y el $13 \%$ de la población en las sociedades occidentales cumplen los criterios requeridos para el diagnóstico de la Fobia Social (FS en adelante) en algún momento de sus vidas (véase Bados, 2009 o Kessler, Berglund, Demler, Jin y Walters, 2005), lo que hace de la FS el tercer trastorno psicológico más frecuente en las sociedades occidentales, tras la depresión y el consumo de alcohol (Ruscio et al., 2008), así como un importante problema de salud mental (Wittchen, Feutsch, Sonntag, Muller y Liebowitz, 2000) y de salud pública (Olivares, 2009), que merma de manera muy significativa la calidad de vida (Hambrick, Turk, Heimberg, Schneier y Liebowitz, 2003) y conlleva importante costes económicos para los sistemas de salud pública (Greenberg et al., 1999; Nardi, 2005). Además, toda esta problemática se ve considerablemente agravada tanto por la elevada comorbilidad del trastorno como por su cronicidad. En relación con la comorbilidad, Kessler, Chiu, Demler y Walters (2005) informaron que los trastornos de ansiedad se asocian con altas tasas de comorbilidad con otros trastornos de ansiedad y depresión que, en su estudio, se sitúan entre el $40 \%$ y el $80 \%$. Respecto de la cronicidad, los datos disponibles permiten sostener que ésta es en general moderadamente estable a lo largo de la vida (véase Rapee y Spence, 2004 o Ruscio et al., 2008).

La eficacia de los tratamientos de la FS ha sido revisada en distintas ocasiones en la última década (así, Barlow, Raffa y Cohen, 2002; García-García, Rosa y Olivares-Olivares, 2011; Jorstad-Stein y Heimberg, 2009; Ponniah y Hollon, 2008 o Salabarría y Echeburúa, 2003), lo que ha permitido a Ollendick y King (2008) concluir que el estudio de este trastorno es una de las áreas en las que la psicología aplicada ha mostrado avances más significativos.

Pese a que ya contamos con distintos estudios que aportan evidencia empírica respecto de la bondad de los tratamientos conductuales y cognitivo-conductuales de la FS en el ámbito adulto, cuando se aplican en contextos controlados y en el desarrollo de la práctica clínica cotidiana, se realice ésta en centros públicos o privados y se aplique individual o grupalmente (véase Gaston, Abbott, Rapee y Neary, 2006 o McEvoy, 2007), todavía están pendiente de solución tanto el problema de los abandonos en la práctica clínica cotidiana (30\%-35\%) como, en muchas ocasiones, el incremento de la duración del tratamiento cuando tiene lugar en el ámbito aplicado (Bados, Balaguer y Saldaña, 2007).

En este contexto, la detección e intervención de personas con FS en el ámbito comunitario educativo puede permitir reducir de manera significativa tanto los abandonos como la duración de los tratamientos. Ello es posible porque este contexto permite utilizar la infraestructura de la institución educativa para llevar a cabo los tratamientos en horarios compatibles con las tareas docentes y de aprendizaje, lo que puede suponer tanto un considerable ahorro de costes como respecto de la duración del tratamiento, al no verse este último afectado por listas de espera o la saturación de los servicios de salud mental de los centros públicos. Ambos beneficios estarían presentes en la detección e intervención en población universitaria.

En un intento de poner a prueba estos supuestos, el presente estudio experimental tiene dos objetivos. El primero es traducir, adaptar y validar para su uso con población española adulta y universitaria (adultos-jóvenes) la Terapia para la Eficacia Social/social effectiveness therapy (Turner, Beidel, y Cooley, 1994), un tratamiento multicomponente cuya eficacia no ha sido todavía probada en población de habla española. El segundo, detectar y tratar entre la población universitaria a adultos-jóvenes que cumpliendo los criterios requeridos para el diagnóstico de FSG (APA, 2000) no hayan recibido ni estén recibiendo tratamiento alguno al respecto.

\section{Método}

\section{Participantes}

Los participantes fueron 31 adultos-jóvenes, estudiantes universitarios de primer curso de la Universidad de Murcia, que cumplían los criterios requeridos por el DSM-IV-TR (APA, 2000) para el diagnóstico de Fobia Social Generalizada (FSG en adelante). La edad media fue de 19,6 años (DE: 0,7; rango: $18-20)$, siendo la mayoría mujeres (64.5\%). Los participantes fueron asignados aleatoriamente a la condición experimental $(n=16)$ y al grupo de control lista de espera $(n=15)$. Ninguno había sido tratado previamente por problemas de tipo psicológico ni estaba recibiendo tratamiento por esta razón. En la Tabla 1 presentamos la distribución de los participantes en cada uno de los grupos, por edad y género, así como otros datos relevantes de la muestra.

\section{Procedimiento}

1) En el proceso de detección se aplicó el SPAI (Social Phobia and Anxiety Inventory; Turner, Beidel, Dancu y 
Tabla 1. Medidas de variables referentes a los participantes.

\begin{tabular}{|c|c|c|c|}
\hline & & & \\
\hline & & $\begin{array}{c}\text { SET (GE) } \\
\quad(\mathrm{n}=16)\end{array}$ & $\begin{array}{l}\text { GCLE } \\
(n=15)\end{array}$ \\
\hline Edad M (DT) & & $19.5(0.7)$ & $19.5(0.6)$ \\
\hline Género & Chicos & $44 \%$ & $27 \%$ \\
\hline & Chicas & $56 \%$ & $73 \%$ \\
\hline NSSTEI M (DT) & & $5.1(1.3)$ & $5.3(1.3)$ \\
\hline NSSTEA M (DT) & & $2.8(0.9)$ & $2.9(1)$ \\
\hline Comorbilidad & Agorafobia & $6.3 \%$ & $2 \%$ \\
\hline & Ataques de pánico & $7.5 \%$ & $6.7 \%$ \\
\hline & Trastorno de ansiedad generalizada & $35 . \%$ & $40 \%$ \\
\hline & Episodio depresivo mayor & $28 \%$ & $20 \%$ \\
\hline & Trastorno distímico & $44 \%$ & $53 \%$ \\
\hline & Abuso del consumo de alcohol & $33 \%$ & $27 \%$ \\
\hline & Abuso del consumo de otras sustancias tóxicas & $1 \%$ & $3 \%$ \\
\hline & Trastorno de personalidad por evitación & $73 \%$ & $67 \%$ \\
\hline & Trastorno obsesivo-compulsivo & $6.25 \%$ & $2.00 \%$ \\
\hline
\end{tabular}

Nota. M: Media, DT: Desviación Típica, NSSTEI = Número de situaciones sociales temidas/evitadas de interacción, NSSTEA = Número de situaciones sociales temidas/evitadas de actuación, $\mathbf{S E T}=$ Social Effectiveness Therapy, $\mathbf{G E}=$ Grupo experimental y $\mathbf{G C L E}=$ Control lista de espera.

Stanley, 1989) para realizar un cribado en el comienzo del primer curso a todos los alumnos de las licenciaturas de Enfermería, Medicina, Psicología y Ciencias de la Educación (Pedagogía y Magisterio) que aceptaron de forma voluntaria $(n=1123)$. Con el fin de maximizar la probabilidad de no incluir falsos positivos y minimizar la de rechazar falsos negativos, seleccionamos aquellos cuya puntuación se encontraba por encima del punto de corte previamente determinado (Olivares, OlivaresOlivares y Macià, 2005). El valor de este punto de corte fue de 97; el 6\% puntuó por encima de $97(n=67)$.

2) A todos los que habían obtenido una puntuación igual o superior al punto de corte en la aplicación del SPAI se les realizó individualmente una entrevista clínica con fines diagnósticos (ADIS-IV: L; Di Nardo, Brown y Barlow, 1994), siguiendo primero el proceso de entrevista recogido en su sección de FS y, cuando el participante cumplía los criterios requeridos, valorando el cumplimiento de los criterios del resto de las secciones. Cumplieron los criterios para el diagnóstico de FS 62 participantes (92.5\%), de los que 37 cumplían los criterios para el diagnóstico de Fobia Social Generalizada-FSG-(60\%) y 25 los de Fobia Social Específica-FSE- (40\%). A estos últimos se les ofreció la opción de recibir tratamiento al margen de la investigación. De los 37 participantes con FSG, 6 decidieron no adscribirse al programa de tratamiento $(16.7 \%)$.

3) Distribuimos aleatoriamente a los participantes a la Condición Experimental (GE en adelante), integrada por dos subgrupos de 8 participantes, y 15 a la condición GCLE.

4) Realizamos la evaluación pretest.

5) Aplicamos el tratamiento a los integrantes del GE.

6) Realizamos la evaluación postest a los integrantes de ambos grupos.

7) Registro de los datos correspondientes a las evaluaciones relativas al seguimiento a los 6 y 12 meses (GE). Por razones éticas, se ofreció a los participantes en el grupo de control-lista de espera (GCLE) la posibilidad de incorporarse a un grupo de tratamiento tras el postest. La evaluación fue realizada por cuatro colaboradores (dos parejas hombre-mujer), entrenados para llevar a cabo la selección, evaluación pre-tratamiento y posttratamiento; una pareja realizó la evaluación pretest y la del seguimiento a los 6 meses, la otra evaluó el postest y el seguimiento a los 12 meses.

\section{Instrumentos}

Todos los participantes cumplimentaron en el pretest y postest los instrumentos que a continuación se enumeran, a su vez, los participantes del GE los cumplimentaron también en el seguimiento a los 6 y 12 meses:

- Anxiety Disorders Interview Schedule for DSM-IV (ADIS-IV:L, Di Nardo, Brown y Barlow, 1994). Se utilizó como entrevista clínica diagnóstica para registrar el número de situaciones sociales de actuación y relación señaladas como temidas/evitadas en su sección de fobia social, las 
cuales se tomaron como variable dependiente para el cálculo de la significación clínica de los efectos de tratamiento. Esta entrevista ha mostrado una excelente fiabilidad test-retest e interjueces en su aplicación a la fobia social.

- $\quad$ Social Phobia and Anxiety Inventory (SPAI; Turner, Beidel, Dancu y Stanley, 1989). Evalúa tres dimensiones: fobia social, agorafobia y puntuación diferencia. Olivares, Olivares-Olivares y Macià (2005) estudiaron sus propiedades psicométricas en población adulta universitaria española, hallando coeficientes de consistencia interna de .95 para la subescala de fobia social, .86 en la subescala de agorafobia, .89 para la puntuación diferencia y una fiabilidad test-retest elevada a las 12 semanas (.88).

- $\quad$ Social Phobia Scale (SPS; Mattick y Clarke, 1998). Diseñada para evaluar la ansiedad de actuación (por ejemplo, llamar por teléfono, comer o beber en público). La consistencia interna fue de .90 en población adolescente española entre 14 y 18 años (García-López, Olivares, Hidalgo, Beidel y Turner, 2000).

- $\quad$ Escala de Ansiedad en la Interacción Social (SIAS; Mattick y Clarke, 1998). Evalúa la ansiedad ante interacciones sociales. En población de lengua inglesa ha mostrado buena consistencia interna y fiabilidad test-retest, así como validez concurrente y discriminante. En un estudio realizado con población adulta española (Olivares, García-López e Hidalgo, 2001), los resultados son consistentes con lo informado. Mattick y Clarke (1998) muestran valores de fiabilidad excelentes.

- Fear of Negative Evaluation Scale (FNES; Watson y Friend, 1969). Evalúa el miedo a la evaluación negativa; consta de 30 ítems con dos posibilidades de respuesta relacionadas con los componentes cognitivos del trastorno. La fiabilidad test-retest, con un mes de intervalo, es de .78 y el Coeficiente Alfa de consistencia interna es de .94 para población española (Salabarría y Echeburúa, 1995).

- $\quad$ La escala Self-statements during public speaking / Autoafirmaciones durante una situación de hablar en público (SSPS/AHP; Hofmann y Di Bartolo, 2000) evalúa las respuestas cognitivas ante la situación de hablar en público y está integrada por dos subescalas: la de autoverbalizaciones positivas y la de autoverbalizaciones negativas. La consistencia interna y la fiabilidad test-retest informadas por Hofmann y Dibartolo es media: $\alpha=.75$ (subescala SSPS-P -autoafirmaciones positivas-), $\alpha=.86$ (subescala- $\mathrm{N}-$ autoafirmaciones negativas-); $r=.78$ en la prueba test-retest a los 3 meses para la subescala SSPS-P $(r=.78)$ y $r=.80$ para la subescala SSPS-N. En una investigación dirigida por los profesores J. Olivares y M. D. Hidalgo (Rivero, 2005), se hallaron los siguientes valores para la consistencia interna: $\alpha=.86$-SSPS-N-; $\alpha=.69$-SSPS-P- y $\alpha=.53$-SSPS-

- Rathus Asertive Scale (RAS; Rathus, 1973). Mide la conducta asertiva del participante. Su coeficiente alfa de consistencia interna varía entre .73 y .86 para población española (Salabarría y Echeburúa, 1995).
- Escala de Inadaptación (EI; Echeburúa, Corral y Fernández-Montalvo, 2000). Construida para evaluar el grado en el que un trastorno puede afectar a diferentes áreas de la vida cotidiana: trabajo, estudios, vida social, tiempo libre, relación de pareja y vida familiar. La fiabilidad medida con el coeficiente alfa de Cronbach en población española es de .94 .

- Cuestionario de Confianza para Hablar en Público (CCHP) de Gilkinson (1942). Se empleó la versión de Paul (1966) con el objeto de evaluar tanto el miedo como la seguridad a hablar en público (antes, durante y después de la actuación). Los resultados con población adolescente española mostraron una consistencia interna alta: 0.89 (García-López, Olivares y Vera-Villarroel, 2003).

\section{Diseño}

Se utilizó un diseño experimental con un factor intra (pretest, postest y seguimiento a los seis y doce meses) y otro inter (grupo de tratamiento SET vs. GCLE). Las dos condiciones experimentales fueron evaluadas antes de iniciarse el tratamiento e inmediatamente después de terminado. Del GE también se obtuvieron datos de seguimiento (seis y doce meses), pero no del GCLE, pues a sus integrantes se les ofreció la posibilidad de iniciar el tratamiento tras la evaluación postest. Las sesiones de intervención se llevaron a cabo en horario de mañana en el campus de la universidad. El tratamiento fue aplicado por dos terapeutas de distinto sexo, con experiencia en el tratamiento de la fobia social. No se produjo mortalidad experimental.

\section{Tratamiento}

El programa utilizado con los integrantes del grupo experimental-tratamiento fue la Terapia para la Eficacia Social (Social Effectiveness Therapy-SET-; Turner, Beidel, Cooley, Woody y Messer, 1994). El tratamiento consta de cuatro componentes interrelacionados: psicoeducación, entrenamiento en habilidades sociales, exposición en vivo e imaginada y práctica programada (autoexposición).

a) Fase educativa (reconceptualización), en la que se les proporciona a los participantes una explicación, desde la perspectiva del modelo conductual, de la ansiedad en general y de la FS en particular (incluidos los patrones de expresión, la relación con la timidez y con otros trastornos de ansiedad); además se les informa sobre el programa de tratamiento. Proporcionamos a los participantes una explicación razonable del porqué de lo que les ocurre y qué dejamos hacer para ayudarles. Esta explicación es necesaria para que puedan comprender nuestra forma de actuar durante el desarrollo de las sesiones, para que conozcan qué se espera de ellos e incrementen sus expectativas sobre los posibles resultados del tratamiento. 
b) Entrenamiento en habilidades sociales. Constituye el formato en el que se inscribe y desarrolla casi todo el tratamiento. Desde una estrategia de aprendizaje estructurado (instrucciones, modelado, ensayo de conducta, refuerzo positivo y retroalimentación), los participantes aprenden y/o practican el modo de iniciar y mantener conversaciones con conocidos, poco conocidos y extraños; a expresarse asertivamente; a dar y recibir cumplidos; a iniciar y mantener amistades; a relacionarse con personas de distinto sexo y relevancia social; a expresarse adecuadamente ante una audiencia, etc. Además, este componente permite exponer controladamente a los participantes de modo tanto individual como en el contexto del grupo, en vivo y en imaginación, a situaciones sociales (discrecionales y simuladas) que les generan respuestas de ansiedad/ evitación social.

c) La exposición en vivo y en imaginación. Es el componente activo más importante en el tratamiento de los problemas de ansiedad en general y de la FS en particular. La exposición se aplicó en sesiones individualizadas, tanto en vivo como en imaginación.

d) Práctica programada. Se llevó a cabo en sesiones individuales. Consiste en la autoexposición, dirigida por el terapeuta, en el ambiente natural. El terapeuta planifica las actividades pero es el paciente el que asume el control, facilitando el incremento de la confianza en sí mismo. El objetivo es consolidar los aprendizajes y generalizarlos a contextos "no seguros".

La distribución del número de sesiones de entrenamiento por semana, su duración y formato fue la que sigue: (i) Fase educativa: una sesión de dos horas de duración, en grupo; (ii) Fase de entrenamiento en habilidades sociales: dos sesiones por semana durante cuatro semanas, en grupo; (iii) Fase de exposición: dos sesiones por semana durante ocho semanas, combinando ocho sesiones de entrenamiento individual y ocho en grupo; (iv) Práctica programada: una sesión semanal de una hora de duración, con formato individual y durante las cuatro últimas semanas.

La (ii), (iii) y (iv) requieren un trabajo adicional de los terapeutas que ronda las 40 horas. La aplicación de la Terapia para la Eficacia Social se desarrolla a lo largo de 16 semanas.

A diferencia de la versión original de la SET, en la que tanto en la fase educativa como en la de entrenamiento en habilidades sociales se trabajó con el formato del pequeño grupo (3-4 participantes/unidad de tratamiento), nosotros lo hemos realizado con 8-10 integrantes/ unidad de tratamiento. Ello se debe a que en estudios piloto, realizados ad hoc, hallamos que el tamaño del grupo que mejores resultados alcanzaba era éste para el rango de edad y características de nuestra muestra.

\section{Resultados}

De acuerdo con el diseño, se realizaron análisis comparativos tanto intergrupo como intragrupo, utilizando para ello el paquete estadístico SPSS.

Respecto a los análisis intergrupo, en primer lugar procedimos a comprobar si existían diferencias en el pretest entre los grupos mediante la realización de comparaciones de medias o frecuencias según el nivel de medida de cada una de las variables para, posteriormente, pasar a comprobar las diferencias en el postest.

La Tabla 2 muestra los resultados en las distintas variables dependientes. En base a estos puede afirmarse que ambos grupos estaban inicialmente equiparados, salvo en las variables que miden la ansiedad de actuación (SPS), la ansiedad social de interacción (SIAS), autoverbalizaciones negativas durante el habla en público (AHP), la autoestima y la asertividad. Por ello, la comparación intergrupo se realizó con la prueba $t$ de Student para todas las variables, menos para estas cinco en las que se utilizó un ANCOVA. En la Tabla 2 también se presenta el tamaño del efecto. Como podemos comprobar, en todas las variables encontramos que se produce una "mejora" del grupo experimental respecto al de control y, si tenemos en cuenta el criterio de Cohen (1988), podemos afirmar que éste es grande en todas las variables.

Respecto al análisis intragrupo, realizamos un análisis de varianza de medidas repetidas en las distintas variables dependientes, cuyos resultados se muestran en la Tabla 3. Como podemos comprobar, en todos los casos el valor $\mathrm{F}$ del ANOVA fue significativo $(p<.05)$ y se procedió al estudio de los efectos principales por el procedimiento de Bonferroni.

La significación clínica de los cambios fue determinada mediante el estudio del porcentaje de participantes que dejaban de cumplir los criterios requeridos para el diagnóstico de FS por el DSM-IV-TR (véase Tabla 4). Es decir, aquellos que presentaban una remisión total del número de situaciones sociales temidas/evitadas e inicialmente medidas, quienes presentaban una remisión parcial de éstas (reducción entre un 75\%-99.99\%; un 50\%-74.99\% y un 0\%-49.99\%).

Centrándonos en la remisión al $100 \%$ en el postest, observamos que no se alcanzaron diferencias significativas entre ambos grupos $(p \leq .05)$, pues pese a no presentarse ninguna remisión del trastorno entre los integrantes del GCLE, ésta solo alcanzó al $18.75 \%$ de los participantes tratados en el GE/SET. (con el transcurrir del tiempo, el número de remisiones completas siguió incrementándose en el GE/SET alcanzando al 56.25\% de sus integrantes en el seguimiento a los seis meses y aumentando ésta hasta el $87.5 \%$ de los integrantes en las medidas de seguimiento tomadas un año después de haber terminado el tratamiento.

En cuanto a la remisión parcial, encontraríamos diferencias estadísticamente significativas $(p \leq .05)$ entre el GE/ SET y el GCLE si sumásemos los dos primeros criterios a 
Tabla 2. Comparaciones inter-grupos.

\begin{tabular}{|c|c|c|c|c|c|c|c|}
\hline \multirow[b]{2}{*}{$\begin{array}{l}\text { Medida de la ansiedad/ } \\
\text { evitación social }\end{array}$} & \multirow[b]{2}{*}{ Momento } & \multicolumn{2}{|c|}{ Grupo } & \multirow[b]{2}{*}{$T$} & \multirow[b]{2}{*}{$\mathrm{F}$} & \multirow[b]{2}{*}{$p$} & \multirow[b]{2}{*}{ TE } \\
\hline & & $\begin{array}{c}\text { SET (GE) }(n=16) \\
\text { M (DT) }\end{array}$ & $\begin{array}{c}\text { GCLE }(\mathrm{n}=15) \\
\mathrm{M}(\mathrm{DT})\end{array}$ & & & & \\
\hline SPAI- Fobia Social & $\begin{array}{l}\text { Pretest } \\
\text { postest }\end{array}$ & $\begin{array}{l}144.31(13.78) \\
100.25(16.62)\end{array}$ & $\begin{array}{l}141.73(17.65) \\
147.00(19.09)\end{array}$ & $\begin{array}{c}.45 \\
-7.28\end{array}$ & & $\begin{array}{l}.652 \\
.000\end{array}$ & 2.62 \\
\hline SPAI-Agorafobia & $\begin{array}{l}\text { Pretest } \\
\text { postest }\end{array}$ & $\begin{array}{l}23.37(6.6) \\
12.94(5.6)\end{array}$ & $\begin{array}{c}22.27(6.88) \\
23.4(6.04)\end{array}$ & $\begin{array}{c}.46 \\
-5.00\end{array}$ & & $\begin{array}{l}.651 \\
.000\end{array}$ & 1.79 \\
\hline SPAI-P. Diferencia & $\begin{array}{l}\text { Pretest } \\
\text { postest }\end{array}$ & $\begin{array}{c}120.94(13.15) \\
87.44(15.11)\end{array}$ & $\begin{array}{l}119.47(13.52) \\
123.53(14.31)\end{array}$ & $\begin{array}{c}.31 \\
-6.82\end{array}$ & & $\begin{array}{l}.761 \\
.000\end{array}$ & 2.45 \\
\hline SPS & $\begin{array}{l}\text { Pretest } \\
\text { postest }\end{array}$ & $\begin{array}{c}62.31(7.33) \\
40.31(12.31)\end{array}$ & $\begin{array}{c}50.8(6.67) \\
55.27(5.89)\end{array}$ & 4.56 & 35.63 & $\begin{array}{l}.000 \\
.000\end{array}$ & 0.72 \\
\hline SIAS & $\begin{array}{l}\text { Pretest } \\
\text { postest }\end{array}$ & $\begin{array}{l}71.18(6.93) \\
43.00(68.4)\end{array}$ & $\begin{array}{l}65.8(5.64) \\
68.4(4.55)\end{array}$ & 2.36 & 34.45 & $\begin{array}{l}.025 \\
.000\end{array}$ & 0.71 \\
\hline CCHP & $\begin{array}{l}\text { Pretest } \\
\text { postest }\end{array}$ & $\begin{array}{l}32.87(16.79) \\
67.62(20.13)\end{array}$ & $\begin{array}{l}35.87(7.99) \\
35.07(9.95)\end{array}$ & $\begin{array}{l}-.64 \\
5.76\end{array}$ & & $\begin{array}{l}.529 \\
.000\end{array}$ & 2.03 \\
\hline $\begin{array}{l}\text { NSSTE } \\
\text { Relación }\end{array}$ & $\begin{array}{l}\text { Pretest } \\
\text { postest }\end{array}$ & $\begin{array}{l}5.12(1.31) \\
1.69(1.44)\end{array}$ & $\begin{array}{c}5.27(1.33) \\
5.4(1.12)\end{array}$ & $\begin{array}{l}-.29 \\
-7.94\end{array}$ & & $\begin{array}{l}.768 \\
.000\end{array}$ & 2.86 \\
\hline $\begin{array}{l}\text { NSSTE } \\
\text { Actuación }\end{array}$ & $\begin{array}{l}\text { Pretest } \\
\text { postest }\end{array}$ & $\begin{array}{l}2.75(0.93) \\
1.06(0.77)\end{array}$ & $\begin{array}{l}2.87(0.99) \\
3.13(0.91)\end{array}$ & $\begin{array}{l}-.34 \\
-6.82\end{array}$ & & $\begin{array}{l}.738 \\
.000\end{array}$ & 2.46 \\
\hline $\begin{array}{l}\text { NSSTE } \\
\text { Total }\end{array}$ & $\begin{array}{l}\text { Pretest } \\
\text { postest }\end{array}$ & $\begin{array}{l}7.88(1.89) \\
2.75(2.05)\end{array}$ & $\begin{array}{l}8.13(1.96) \\
8.53(1.77)\end{array}$ & $\begin{array}{l}-.37 \\
-8.39\end{array}$ & & $\begin{array}{l}.712 \\
.000\end{array}$ & 3.01 \\
\hline AHP & $\begin{array}{l}\text { Pretest } \\
\text { postest }\end{array}$ & $\begin{array}{l}36.81(6.02) \\
19.37(9.36)\end{array}$ & $\begin{array}{c}22.8(10.06) \\
24.2(9.74)\end{array}$ & 4.74 & 19.52 & $\begin{array}{l}.000 \\
.000\end{array}$ & 0.58 \\
\hline FNES & $\begin{array}{l}\text { Pretest } \\
\text { postest }\end{array}$ & $\begin{array}{l}27.25(1.95) \\
18.00(3.98)\end{array}$ & $\begin{array}{l}27.27(2.19) \\
28.07(1.58)\end{array}$ & $\begin{array}{l}-.02 \\
-9.36\end{array}$ & & $\begin{array}{l}.982 \\
.000\end{array}$ & 3.28 \\
\hline EI & $\begin{array}{l}\text { Pretest } \\
\text { postest }\end{array}$ & $\begin{array}{l}31.25(3.13) \\
22.25(5.74)\end{array}$ & $\begin{array}{c}29.33(3.72) \\
30.2(3.07)\end{array}$ & $\begin{array}{c}1.56 \\
-4.84\end{array}$ & & $\begin{array}{l}.130 \\
.000\end{array}$ & 1.71 \\
\hline RAS & $\begin{array}{l}\text { Pretest } \\
\text { postest }\end{array}$ & $\begin{array}{c}-45.25(18.93) \\
-4.19(19.87)\end{array}$ & $\begin{array}{l}-29.27(14.09) \\
-31.13(13.11)\end{array}$ & -2.65 & 32.75 & $\begin{array}{l}.013 \\
.000\end{array}$ & 0.70 \\
\hline
\end{tabular}

SET $=$ Social Effectiveness Therapy, GE = Grupo experimental, GCLE = Control Lista de Espera, M: Media, DT: Desviación Típica, $\mathbf{n}$ : Tamaño del grupo, $\mathbf{F}=$ F de Snedecor, $\mathbf{t}=\mathrm{t}$ de Student, SPAI-Fobia Social= Inventario de Ansiedad y Fobia Social. Subescala de Fobia Social, SPAI-Diferencia $=$ Inventario de Ansiedad y Fobia Social, Puntuación de diferencia, SPAI-Agorafobia= Inventario de Ansiedad y Fobia Social. Subescala de Agorafobia, SPS = Social Phobia Scale, SIAS = Escala de Ansiedad en la Interacción Social, CCHP = Cuestionario de Confianza para Hablar en Público, NSSTE Relación = Número de situaciones sociales temidas/evitadas de relación, NSSTE Actuación = Número de situaciones sociales temidas/evitadas de actuación, NSSTE Total = Total del número de situaciones sociales temidas/evitadas, $\mathbf{A H P}=$ Cuestionario de Autoverbalizaciones al Hablar en Público, FNE = Fear of Negative Evaluation Scale, EI = Escala de Inadaptación, RAS $=$ Rathus Asertive Scale, TS/CO = Test situacional/Contacto Ocular y TE $=$ Tamaño del efecto.

Tabla 3. Resultados de la comparación intra-grupo.

\begin{tabular}{|c|c|c|c|c|c|c|c|}
\hline \multirow{2}{*}{$\begin{array}{l}\text { Medidas de la ansiedad/ } \\
\text { evitación social }\end{array}$} & \multirow[b]{2}{*}{ Momento } & \multirow{2}{*}{$\begin{array}{c}\text { SET (GE) }(\mathrm{n}=16) \\
\mathrm{M}(\mathrm{DT})\end{array}$} & \multirow[b]{2}{*}{$\mathrm{F}$} & \multirow[b]{2}{*}{$\mathrm{p}$} & \multirow[b]{2}{*}{ TE } & \multirow[b]{2}{*}{ Comparaciones } & \multirow[b]{2}{*}{$\mathrm{p}$} \\
\hline & & & & & & & \\
\hline \multirow{6}{*}{ SPAI- Fobia Social } & Pretest & $145.33(13.62)$ & 119.14 & .000 & 0.895 & Pretest-Postest & .000 \\
\hline & Postest & $101.13(16.81)$ & & & & Pretest-Seg 6 & .000 \\
\hline & Segui. 6 & $76.53(19.84)$ & & & & Pretest-Seg12 & .000 \\
\hline & Segui.12 & $61.07(22.54)$ & & & & Post-Seg 6 & .000 \\
\hline & & & & & & Post-Seg 12 & .000 \\
\hline & & & & & & Seg6-Seg12 & .013 \\
\hline
\end{tabular}


Continuación Tabla 3

\begin{tabular}{|c|c|c|c|c|c|c|c|}
\hline \multirow{2}{*}{$\begin{array}{l}\text { Medidas de la ansiedad/ } \\
\text { evitación social }\end{array}$} & \multirow[b]{2}{*}{ Momento } & \multirow{2}{*}{$\frac{\text { SET }(\mathrm{GE})(\mathrm{n}=16)}{\mathrm{M}(\mathrm{DT})}$} & \multirow[b]{2}{*}{$\mathrm{F}$} & \multirow[b]{2}{*}{$\mathrm{p}$} & \multirow[b]{2}{*}{$\mathrm{TE}$} & \multirow[b]{2}{*}{ Comparaciones } & \multirow[b]{2}{*}{$\mathrm{p}$} \\
\hline & & & & & & & \\
\hline \multirow{6}{*}{ SPAI-Agorafobia } & Pretest & $23.67(6.72)$ & 54.32 & .000 & 0.795 & Pretest-Postest & .000 \\
\hline & Postest & $13.07(5.77)$ & & & & Pretest-Seg 6 & .000 \\
\hline & Segui. 6 & $6.93(3.47)$ & & & & Pretest-Seg12 & .000 \\
\hline & Segui.12 & $5.67(7.16)$ & & & & Post-Seg 6 & .000 \\
\hline & & & & & & Post-Seg 12 & .002 \\
\hline & & & & & & Seg6-Seg12 & 1.00 \\
\hline \multirow{6}{*}{ SPAI-Diferencia } & Pretest & $121.67(13.27)$ & 78.54 & .000 & 0.849 & Pretest-Postest & .000 \\
\hline & Postest & $88.2(15.32)$ & & & & Pretest-Seg 6 & .000 \\
\hline & Segui. 6 & $69.6(18.13)$ & & & & Pretest-Seg12 & .000 \\
\hline & Segui.12 & $56.07(19.41)$ & & & & Post-Seg 6 & .000 \\
\hline & & & & & & Post-Seg 12 & .000 \\
\hline & & & & & & Seg6-Seg12 & .017 \\
\hline \multirow{6}{*}{ SPS } & Pretest & $62.53(7.53)$ & 80.55 & .000 & 0.852 & Pretest-Postest & .000 \\
\hline & Postest & $41.27(12.11)$ & & & & Pretest-Seg 6 & .000 \\
\hline & Segui. 6 & $30.07(12.53)$ & & & & Pretest-Seg12 & .000 \\
\hline & Segui.12 & $22.47(12.65)$ & & & & Post-Seg 6 & .000 \\
\hline & & & & & & Post-Seg 12 & .000 \\
\hline & & & & & & Seg6-Seg12 & .026 \\
\hline \multirow{6}{*}{ SIAS } & Pretest & 71.07(7.15) & 87.72 & .000 & 0.858 & Pretest-Postest & .000 \\
\hline & Postest & $44.53(14.40)$ & & & & Pretest-Seg 6 & .000 \\
\hline & Segui. 6 & $30.8(14.64)$ & & & & Pretest-Seg12 & .000 \\
\hline & Segui.12 & $24.2(14.96)$ & & & & Post-Seg 6 & .000 \\
\hline & & & & & & Post-Seg 12 & .001 \\
\hline & & & & & & Seg6-Seg12 & .160 \\
\hline \multirow{6}{*}{ CCHP } & Pretest & $34.07(16.66)$ & 116.07 & .000 & 0.892 & Pretest-Postest & .000 \\
\hline & Postest & $66.6(20.4)$ & & & & Pretest-Seg 6 & .000 \\
\hline & Segui. 6 & $89.47(19.41)$ & & & & Pretest-Seg12 & .000 \\
\hline & Segui.12 & $104.07(22)$ & & & & Post-Seg 6 & .000 \\
\hline & & & & & & Post-Seg 12 & .000 \\
\hline & & & & & & Seg6-Seg12 & .002 \\
\hline \multirow{6}{*}{ NSSTE Relación } & Pretest & $5.2(1.32)$ & 132.45 & .000 & 0.904 & Pretest-Postest & .000 \\
\hline & Postest & $1.8(1.42)$ & & & & Pretest-Seg 6 & .000 \\
\hline & Segui. 6 & $0.4(0.83)$ & & & & Pretest-Seg12 & .000 \\
\hline & Segui.12 & $0.4(1.05)$ & & & & Post-Seg 6 & .000 \\
\hline & & & & & & Post-Seg 12 & .002 \\
\hline & & & & & & Seg6-Seg12 & 1.00 \\
\hline \multirow{4}{*}{ NSSTE Actuación } & Pretest & $2.8(0.94)$ & 48.1 & .000 & 0.775 & Pretest-Postest & .000 \\
\hline & Postest & $1.13(0.74)$ & & & & Pretest-Seg 6 & .000 \\
\hline & Segui. 6 & $0.47(0.51)$ & & & & Pretest-Seg12 & .000 \\
\hline & Segui.12 & $0.2(0.77)$ & & & & Post-Seg 6 & .019 \\
\hline \multirow{8}{*}{ NTSSTE } & & & & & & Post-Seg 12 & .007 \\
\hline & & & & & & Seg6-Seg12 & 1.00 \\
\hline & Pretest & $8(1.89)$ & 143.63 & .000 & 0.911 & Pretest-Postest & .000 \\
\hline & Postest & $2.93(1.98)$ & & & & Pretest-Seg 6 & .000 \\
\hline & Segui. 6 & $0.87(1.19)$ & & & & Pretest-Seg12 & .000 \\
\hline & Segui.12 & $0.53(1.81)$ & & & & Post-Seg 6 & .000 \\
\hline & & & & & & Post-Seg 12 & .000 \\
\hline & & & & & & Seg6-Seg12 & 1.00 \\
\hline
\end{tabular}


Continuación Tabla 3

\begin{tabular}{|c|c|c|c|c|c|c|c|}
\hline \multirow{2}{*}{$\begin{array}{l}\text { Medidas de la ansiedad/ } \\
\text { evitación social }\end{array}$} & \multirow[b]{2}{*}{ Momento } & $\operatorname{SET}(\mathrm{GE})(\mathrm{n}=16)$ & \multirow[b]{2}{*}{$\mathrm{F}$} & \multirow[b]{2}{*}{$\mathrm{p}$} & \multirow[b]{2}{*}{$\mathrm{TE}$} & \multirow[b]{2}{*}{ Comparaciones } & \multirow[b]{2}{*}{$\mathrm{p}$} \\
\hline & & M (DT) & & & & & \\
\hline \multirow[t]{6}{*}{ AHP } & Pretest & $36.4(5.99)$ & 97.32 & .000 & 0.874 & Pretest-Postest & .000 \\
\hline & Postest & $20.2(9.06)$ & & & & Pretest-Seg 6 & .000 \\
\hline & Segui. 6 & $10.2(8.91)$ & & & & Pretest-Seg12 & .000 \\
\hline & Segui.12 & $6.87(9.02)$ & & & & Post-Seg 6 & .000 \\
\hline & & & & & & Post-Seg 12 & .000 \\
\hline & & & & & & Seg6-Seg12 & .020 \\
\hline \multirow[t]{6}{*}{ FNES } & Pretest & $27.2(2.01)$ & 82.32 & .000 & 0.855 & Pretest-Postest & .000 \\
\hline & Postest & $18.4(3.77)$ & & & & Pretest-Seg 6 & .000 \\
\hline & Segui. 6 & $13.87(3.64)$ & & & & Pretest-Seg12 & .000 \\
\hline & Segui.12 & $12.4(4.97)$ & & & & Post-Seg 6 & .000 \\
\hline & & & & & & Post-Seg 12 & .002 \\
\hline & & & & & & Seg6-Seg12 & .906 \\
\hline EI & Pretest & $31.4(3.18)$ & 117.04 & .000 & 0.893 & Pretest-Postest & .000 \\
\hline \multirow[t]{5}{*}{ Adaptación } & Postest & $22.73(5.6)$ & & & & Pretest-Seg 6 & .000 \\
\hline & Segui. 6 & $15.2(5.02)$ & & & & Pretest-Seg12 & .000 \\
\hline & Segui.12 & $12.8(5.987)$ & & & & Post-Seg 6 & .000 \\
\hline & & & & & & Post-Seg 12 & .000 \\
\hline & & & & & & Seg6-Seg12 & 0.02 \\
\hline RAS & Pretest & $-44.2(19.11)$ & 103.42 & .000 & 0.881 & Pretest-Postest & .000 \\
\hline \multirow[t]{5}{*}{ Asertividad } & Postest & $-4.73(20.45)$ & & & & Pretest-Seg 6 & .000 \\
\hline & Segui. 6 & $11.8(19.45)$ & & & & Pretest-Seg12 & .000 \\
\hline & Segui.12 & $21.67(22 ., 56)$ & & & & Post-Seg 6 & .001 \\
\hline & & & & & & Post-Seg 12 & .000 \\
\hline & & & & & & Seg6-Seg12 & .013 \\
\hline
\end{tabular}

SET $=$ Social Effectiveness Therapy, GE $=$ Grupo experimental, M: Media, DT: Desviación Típica, $\mathbf{n}:$ Tamaño del grupo, F = F de Snedecor, SPAI-Fobia Social $=$ Inventario de Ansiedad y Fobia Social. Subescala de Fobia Social, SPAI-Diferencia $=$ Inventario de Ansiedad y Fobia Social. Puntuación de diferencia, SPAI-Agorafobia= Inventario de Ansiedad y Fobia Social. Subescala de Agorafobia, SPS $=$ Social Phobia Scale, SIAS = Escala de Ansiedad en la Interacción Social, CCHP = Cuestionario de Confianza para Hablar en Público, NSSTE Relación= Número de situaciones sociales temidas/evitadas de relación, NSSTE Actuación = Número de situaciones sociales temidas/evitadas de actuación, NTSSTE $=$ Total del número de situaciones sociales temidas/evitadas, AHP $=$ Cuestionario de Autoverbalizaciones al Hablar en Público, FNE = Fear of Negative Evaluation Scale, EI = Escala de Inadaptación, RAS = Rathus Asertive Scale, $\mathbf{T S} / \mathbf{C O}=$ Test situacional/Contacto Ocular y $\mathbf{T E}=$ Tamaño del efecto.

Tabla 4. Estudio de la significación clínica de los efectos del tratamiento.

\begin{tabular}{|c|c|c|c|c|}
\hline \multirow[b]{2}{*}{ Pos-test } & \multirow{2}{*}{$\begin{array}{l}\text { Remisión } \\
\text { completa }\end{array}$} & \multicolumn{3}{|c|}{ Remisión parcial } \\
\hline & & $75 \%-99.99 \%$ & $50 \%-74.99 \%$ & $0 \%-49.99 \%$. \\
\hline SET (GE) & $3(18.5 \%)$ & $2(12.5 \%)$ & $8(50 \%)$ & $3(18.75 \%)$ \\
\hline Grupo Control & 0 & 0 & 0 & 15 \\
\hline \multicolumn{5}{|c|}{ Seguimiento 6 meses } \\
\hline SET (GE) & $9(56.25 \%)$ & $6(37.5 \%)$ & $1(6.25 \%)$ & 0 \\
\hline \multicolumn{5}{|c|}{ Seguimiento 12 meses } \\
\hline SET (GE) & $14(87.5 \%)$ & $1(6.25 \%)$ & 0 & $1(6.25 \%)$ \\
\hline
\end{tabular}

SET $=$ Social Effectiveness Therapy y $\mathbf{G E}=$ Grupo experimental.

la remisión total, en el postest, dado que ningún participante del grupo control disminuyó en el número de situaciones sociales temidas/evitadas medidas, mientras que, en el grupo tratado, los porcentajes de disminución de situaciones fóbicas se sitúan en $12.5 \%$ (para el criterio 75\%-99.99\%), 50\% (para el criterio 50\%-74.99\%) y $18.75 \%$ (para el criterio 0\%-49.99\%). (Estos porcentajes se sitúan en 37.35\%) 6.25\% y $0 \%$ respectivamente, para el seguimiento a seis meses, y $6.25 \%, 0 \%$ y $6.25 \%$ en el seguimiento a doce meses. 


\section{Discusión}

El primero de los objetivos del presente trabajo fue traducir, adaptar y validar para su aplicación en población española adulta (adultos-jóvenes), que cumple los criterios para el diagnóstico de FSG (APA, 2000), el programa de tratamiento Terapia para la eficacia Social (Turner, Beidel y Cooley, 1994), el cual había mostrado ya su eficacia en el tratamiento de población adulta con este trastorno (Turner, Beidel, Cooley, Woody y Messer, 1994) así como la estabilidad temporal de sus efectos (Turner, Beidel y Cooley, 1995). Nuestros resultados confirman para la adaptación que hemos realizado tanto la eficacia del programa para tratar a adultos jóvenes con FSG, de habla española, como la estabilidad de sus efectos en las medidas de seguimiento realizadas a los 6 y 12 meses de terminada su aplicación.

Como puede observarse en las Tablas 2 y 3 , los resultados muestran una disminución estadísticamente significativa en todas las medidas de las respuestas de ansiedad y evitación social, así como en las de sus correlatos, tanto frente al grupo de control como en las medidas de seguimiento. Ello coincide con los resultados obtenidos en traducción y adaptación de este programa para su aplicación en población adolescente de habla española, tanto a corto y mediano plazo (Olivares, García-López, Beidel y Turner, 1998; Olivares y García-López, 2002) como a largo (García-López et al., 2006), así como con los informados por Olivares, Rosa, Olivares-Olivares y Rosa-Alcázar (2009) en la aplicación de la intervención en adultos jóvenes con fobia social.

Los tamaños del efecto obtenidos, de acuerdo con los criterios de Cohen (1988), también corroboran la bondad de la Terapia para la Eficacia Social. Así, salvo excepción, en las comparaciones intergrupo (véase Tabla 2) éstos son altos en 9 de las 13 variables dependientes y medios en las 4 restantes. Es decir, en ninguna variable el tamaño del efecto ha sido bajo. Destacan especialmente los tamaños del efecto producidos en las variables FNES $(T E=3.28)$, Número total de situaciones sociales temidas/evitadas (TE $=3.01)$ y el relativo a la Subescala de Fobia Social del SPAI $(T E=2.62)$. En los intragrupo (véase tabla 3$)$, todos los tamaños del efectos son altos (TE .8), salvo en la Subescala de Agorafobia del SPAI $(T E=.795)$ y el del Número de situaciones sociales de actuación temidas/evitadas $(T E=.775)$.

Además, en relación con la significación clínica, los resultados también constatan otro hecho reiteradamente documentado: el incremento progresivo de la mejora una vez concluida la aplicación del tratamiento conductual, es decir, en las medidas de seguimiento de los efectos del tratamiento (véase, por ejemplo, a Hunt y Andrews, 1998; Méndez, Sánchez y Moreno, 2001; Rapee, 1993 o Salabarrría y Echeburúa, 2003). Como podemos ver en la Tabla 4, en la medida postest solo alcanzan la remisión completa de la FSG el $18.5 \%$ de los participantes del grupo de tratamiento, dejando de cumplir los criterios requeridos para su diagnóstico; en las medidas del seguimiento realizado a los 6 meses, este porcentaje es ya del $56.25 \%$ y en las realizadas a los 12 meses alcanza el $87.5 \%$.

Estos resultados, en línea con lo sostenido por Bados (2009), entendemos que han de interpretarse en base a razones tales como que el entrenamiento en habilidades sociales facilita la exposición a las situaciones sociales, fomenta el empleo de habilidades sociales infrautilizadas y aumenta en los participantes las expectativas de resultado y de autoeficacia.

Asímismo, los resultados ponen de manifiesto la adecuación del diseño de intervención elegido para intentar lograr nuestro segundo objetivo. En relación con éste, es decir, detectar y tratar entre la población universitaria a adultos-jóvenes que cumplieran los criterios requeridos para el diagnóstico de FSG (APA, 2000), los resultados también confirman que la estrategia utilizada resulta adecuada tanto para reducir la elevada tasa de abandonos que se constatan en la práctica cotidiana de la clínica (30\%-35\% -Bados, Balaguer y Saldaña, 2007-) como para controlar que la duración del tratamiento no exceda de lo planificado, alargando el intervalo de tiempo entre sesiones y con él la probabilidad de abandono; en relación con lo primero nuestra tasa de abandonos ha sido nula y respecto de lo segundo la duración del tratamiento fue la indicada por el programa. Así, entendemos que ello ha sido posible porque el contexto comunitario nos ha permitido utilizar la infraestructura de la institución educativa para llevar a cabo los tratamientos en horarios compatibles con las tareas docentes y de aprendizaje, lo que puede suponer tanto un considerable ahorro de costes como respecto de la duración del tratamiento, al no verse este último afectado, tal como indicábamos en nuestra introducción, por listas de espera o por la saturación de los servicios de salud mental de los centros públicos.

El presente estudio tiene varias limitaciones relativas al tamaño de la muestra, a la validez externa, a la validez ecológica y a la validez interna que es preciso tener en cuenta. Así, en primer lugar, el tamaño de los grupos de nuestra investigación no cumple con las sugerencias del grupo Task Force (1995). En segundo lugar, hemos de indicar que la validez externa de nuestro trabajo está limitada al rango de edad de la muestra y a la población estudiante universitaria de una zona de España, la Región de Murcia. En cuanto a la validez interna, constituye una limitación a nuestro estudio el hecho de que no han sido controladas las variables relativas a la hora del día en la que se aplicó la Terapia para la Eficacia Social. Pese a que fue en horario de mañana y entre las 9 y las 13 horas, no siempre pudo ser a la misma hora; otra limitación a la validez interna se halla en el hecho de la potencial aparición de respuestas de escape/ evitación encubiertas que no fueron controladas. Por último, indicar que la presente investigación no cuenta con datos relativos a la validez social de los cambios ni derivados de 
pruebas observacionales, carencias que también constituyen limitaciones a sus resultados.

Todas las limitaciones mencionadas constituyen parte de las mejoras que habrá que ir introduciendo en las futuras investigaciones, para poner a prueba esta modalidad de tratamiento bajo condiciones de más y mejor control experimental, con el fin de que podamos contar con las garantías suficientes para poder aplicar este programa de tratamiento en población adulta de lengua española en las mejores condiciones posibles.

\section{Referencias}

American Psychiatric Association (2000). Diagnostic and statiscal manual of mental disorders (cuarta edición, texto revisado). Washington, DC: Autor (trad. cast.: DSM-IV-TR: Manual diagnóstico y estadístico de los trastornos mentales. Barcelona: Masson, 2002).

Bados, A. (2009). Fobia social. Facultad de Psicología. Departament de Personalitat, Avaluaciò i Tractaments Psicològics. Universitad de Barcelona. Disponible desde http://diposit.ub.edu/dspace/bitstream/2445/6321/1/Fobia\%20social.pdf

Bados, A., Balaguer, G., y Saldaña, C. (2007). Outcome of congitivebehavioural therapy in training practice with anxiety disorders patients. British Journal of Clinical Psychology, 46, 429-435.

Barlow, D.H, Raffa, S.D., y Cohen, E.M. (2002). Psychosocial treatments for panic disorders, phobias, and genelaized anxiety disorder. En P. Nathan y J. Gorman (Eds.), A guide to treatments that work ( $2^{\mathrm{a}}$ ed., pp. 301-335). Nueva York: Oxford University Press.

Cohen, J. (1988). Statistical power analysis for the behavioral sciences ( $2^{\mathrm{a}}$ ed.). Hillsdale, NY: Lawrence Erlbaum Associated.

Di Nardo, P.A., Brown, T.A., y Barlow, D.H. (1994). Anxiety Disorders Interview Schedule for DSM-IV (Lifetime version). San Antonio, TX: Psychological Corporation.

Echeburúa, E., Corral, P., y Fernández-Montalvo, J. (2000). Escala de Inadaptación (EI): propiedades psicométricas en contextos clínicos. Análisis y Modificación de Conducta, 23, 325-340

García-García, S.E., Rosa, A. I., y Olivares-Olivares, P. J. (2011). Terapia de Exposición Mediante Realidad Virtual e Internet en el Trastorno de Ansiedad/Fobia Social: Una Revisión Cualitativa. Terapia psicológica, 29, 233-243.

García-López, L.J., Olivares, J., Beidel, D., Albano, A.M., Turner, S., y Rosa, A.I. (2006). Efficacy of three treatment protocols for adolescents with social anxiety disorder: a 5-year follow-up assessment. Journal of Anxiety Disorders, 20, 175-191.

García-López, L.J., Olivares, J., y Vera-Villarroel, P.E. (2003). Fobia social: revisión de los instrumentos de evaluación validados para población de lengua española. Revista Latinoamericana de Psicología, 35, 151-160.

Gaston, J.E., Abbott, M.J., Rapee, R.M., y Neary, S.A. (2006). Do empirical supported treatments generalize to private practice?. A benchmarck study of a cognitive-behavioural group of treatment programme for social phobia. British Journal of Clinical Psychology, 45, 33-48.

Gilkinson, H. (1942). Social fears as reported by students in college speech classes. Speech Monographs, 9, 141-160.

Greenberg, P. E., Sisitsky, T., Kessler, R C., Finkelstein, S.N., Berndt, E.R., Davidson, J. R.T., Ballenger, J.C., y Fyer, A. J. (1999). The economic burden of anxiety disorders in the 1990s. Journal of Clinical Psychiatry, 60, 427-435.

Hambrick, J.P., Turk, L. C., Heimberg, R.G., Schneier, F.R., y Liebowitz, M.R. (2003). The experience of disability and quality of life in social anxiety disorder. Depression and Anxiety, 18, 46-50.

Hunt, C., y Andrews, G. (1998). Long-term outcome of panic disorder and social phobia. Journal of Anxiety Disorders, 12, 395-406.

Jorstad-Stein, E.C., y Heimberg, R.G. (2009). Social Phobia: An Update on Treatment. Psychiatric Clinics of North America, 32, 641-663.
Kessler, R.C., Berglund, P., Demler, O., Jin, R., y Walters, E.E. (2005). Lifetime prevalence and age-of-onset distributions of DSM-IV disorders in the national comorbidity survey replication. Archives of General Psychiatry, 62, 593-602.

Kessler, R. C., Chiu, W. T., Demler, O., y Walters, E. (2005). Prevalence, severity, and comorbidity of 12-month DSM-IV disorders in the National Comorbidity Survey Replication. Archives of General Psychiatry, 62, 617-627.

Mattick, R.P., y Clarke, J.C. (1998). Development and validation of measures of social phobia scrutiny fear and social interaction anxiety. Behaviour Research and Therapy, 36, 455-470.

McEvoy, P. M. (2007). Effectiveness of cognitive behavioural group therapy for social phobia in a community clinic: a benchmarcking study. Behavior Research and Therapy, 45, 3030-3040.

Méndez, F.X., Sánchez, J., y Moreno, P. (2001). Eficacia de los tratamientos psicológicos para la Fobia Social: un estudio meta-analítico. Psicología Conductual, 9, 39-59.

Nardi, A.E. (2005). Early diagnosis can decrease the social and economic burden of social anxiety disorder. Australian and New Zeland Journal of Psychiatry, 39, 641-642.

Olivares, J. (2009). Evaluación y tratamiento de la fobia social en la población infanto-juvenil de habla española y portuguesa. Anuario de Psicología, 40, 7-21.

Olivares, J., y García-López, L.J. (2002). Aplicación de la versión española de la Terapia para la Eficacia Social en adolescentes (SET-Asv) al tratamiento de un adolescente con Fobia Social generalizada. Psicología Conductual, 2, 409-419.

Olivares, J., García-López, L.J., Beidel, D.C., y Turner, S.M. (1998). Social Effectiveness Therapy for Adolescents. Spanish Versión (SET-A $)$. (Manuscrito no publicado).

Olivares, J., García-López, L.J., Beidel, D.C., Turner, S.M., Albano, A.M., e Hidalgo, M.D. (2002). Results at long-term among three psychological treatments for adolescents with generalized social phobia (I): Statiscal significance. Psicología Conductual, 10, 147-164.

Olivares, J., Olivares-Olivares, P.J., y Macià, D. (2005). Estudio sobre los datos normativos del "Social Phobia and Anxiety Inventory" (SPAI) en su aplicación a adultos jóvenes españoles. Departamento de Personalidad, Evaluación y Tratamientos Psicológicos. Facultad de Psicología. Universidad de Murcia (Manuscrito no publicado).

Olivares, J., Rosa, A.I., Olivares-Olivares, P.J., y Rosa-Alcázar, A. (2009). Treatment of Young Adults with Generalized Social Phobia. International Journal of Hispanic Psychology, 1, 1-14

Ollendick, T.H., y King, N.J. (2008). Evidence-based treatments for children and adolescents with phobic and anxiety disorders: Issues and commentary. Psicología Conductual/Behavioral Psychology, 16, 365-387.

Paul, G. (1966). Insight vs. Desensibilization in psychotherapy. Stanford, CA: Stanford University Press.

Ponniah, K., y Hollon, S. (2008). Empirically supported psychological interventions for social phobia in adults: a qualitative review of randomized controlled trials. Psychological Medicine, 38, 3-14.

Rapee, R.M. (1993). Recent advances in the treatment of social phobia. Australian Psychologist, 28, 168-171.

Rapee, R.M., y Spence, S. H. (2004). The etiology of social phobia: Empirical evidence and an initial model. Clinical Psychology Review, 24, 737-767.

Rathus, S.A. (1973). 30-items schedule for assessing assertive behavior. Behavior Therapy, 4, 398-406.

Rivero, R. (2005). Validación de tres instrumentos para la medida de la fobia social en la adolescencia: SPS, SIAS y SPSS (Tesis doctoral no publicada). Salamanca: Facultad de Psicología.

Ruscio, A. M., Brown, T. A., Chiu, W. T., Sareen, J., Stein, M. B., y Kessler, R. C. (2008). Social fears and social phobia in the USA: results from the National Comorbidity Survey Replication. Psychological Medicine, 38, 15-28.

Salabarría, K., y Echeburúa, E. (1995). Tratamiento psicológico de la fobia social: un estudio experimental. Análisis y Modificación de Conducta, $21,151-179$. 
Salabarría, K., y Echeburúa, E. (2003). Guía de tratamientos psicológicos eficaces para la Fobia Social. En M. Pérez-Alvares, J.R. FernándezHermida, C. Fernández-Rodríguez e I. Amigo (Eds.), Guía de tratamientos psicológicos eficaces I. Adultos (pp. 271-285). Madrid: Pirámide.

Task Force on Promotion and Dissemination of Psychological Procedures (1995). Training and dissemination of empirically-validated psychological treatments: Report and recommendations. The Clinical Psychologist, 48, 3-23.

Turner, S.M., Beidel, D.C., y Cooley, M.R. (1994). Social Effectiveness Therapy: A program for overcoming social anxiety and social phobia. Mt. Pleasant, SC: Turndel.

Turner, S.M., Beidel, D.C., Cooley, M.R. (1995). Two-years follow-up of social phobics treated with Social Effectiveness Therapy. Behavioral Research and Therapy, 33, 553-555.
Turner, S.M., Beidel, D.C., Cooley, M.R., Woody, S.R., y Messer, S.C. (1994). A multicomponent behavioral treatment for social phobia: Social effectiveness therapy. Behavioral Research and Therapy, 32, 381-390. Turner, S.M., Beidel, D.C., Dancu, C.V., y Stanley, M.A. (1989). An empirically derived inventory to measure social fears and anxiety: The Social Phobia and Anxiety Inventory. Psychological Assessment, 1, 35-40.

Watson, D., y Friend, R. (1969). Measurement of social-evalutive anxiety. Journal of Consulting and Clinical Psychology, 33, 448-457.

Wittchen, H.U., Feutsch, M., Sonntag, H., Muller, N., y Liebowitz, M. (2000). Disability and quality of life in pure and comorbid social phobia: findings from a controlled study. European Psychiatry, 15, 46-58. 
